\title{
The Gluon Exchange Model for diffractive and inelastic collisions
}

\author{
Marek Jeżabek $^{a, *}$ and Andrzej Rybicki ${ }^{a}$ \\ ${ }^{a}$ Institute of Nuclear Physics, Polish Academy of Sciences, \\ Radzikowskiego 152, 31-342 Kraków, Poland \\ E-mail: marek.jezabek@ifj.edu.pl, andrzej.rybicki@ifj.edu.pl
}

We propose a new model for a homogeneous description of hadron-hadron and hadron-nucleus collisions, the Gluon Exchange Model (GEM). While technically our model can be regarded as a generalization of the Dual Parton Model by Capella and Tran Thanh Van, it is fundamentally based on the number of exchanged color octets (gluons) and significantly extends the Fock space of states available for the participating protons and nucleons.

In proton-proton collisions we provide an exact description of the final state proton and neutron spectrum. What is remarkable is that unlike the original DPM, GEM successfully describes the proton "diffractive peak" at high $x_{F}$ as a specific case of color octet exchange.

In proton-nucleus reactions we find that the projectile proton diquark, composed of two valence quarks, cannot survive in more than about half of multiple proton-nucleon processes. Consequently it must be very frequently disintegrated, leading to long transfers of baryon number over rapidity space.

\footnotetext{
*** The European Physical Society Conference on High Energy Physics (EPS-HEP2021), ***

*** 26-30 July $2021 * * *$

*** Online conference, jointly organized by Universität Hamburg and the research center DESY ***
}

\footnotetext{
${ }^{*}$ Speaker
} 


\section{Introduction: the Gluon Exchange Model, or solving the puzzle of nuclear stopping power}

This paper will be concerned with "soft" (non-perturbative) proton-proton and proton-nucleus collisions at a relatively moderate energy of $\sqrt{s} \simeq 20 \mathrm{GeV}$ in the nucleon-nucleon center-of-mass system. The implications of the phenomenological studies performed therein touch all the "high" energy scale including the LHC and cosmic regimes, in particular also nucleus-nucleus ("heavy ion") physics.

The main problem which this study will address is the following:

- in a non-perturbative proton-nucleus (pA) collision, how does the proton encode knowledge on the number of nucleons which it collides with?

The question above we define as "the puzzle of nuclear stopping power", an issue with a long record of research [1-8] which we will not stress here. In our paper [9] we remind some of the arguments and difficulties encountered therein, in particular the internal contradictions inherent to the sequential description which preclude encoding this information directly in the projectile energy, as well as the failure of the original Dual Parton Model $[5,10]$ in describing the "nuclear stopping power" estimated on the basis of Fermilab data [3,6]. Improvements of the latter model in terms of the string junction mechanism by Rossi and Veneziano [11] were described elsewhere [7, 8]. In our paper [12] we proposed an independent approach (GEM, the Gluon Exchange Model) where the latter encoding occurs through the partonic structure of the proton at the space-time moment of the collision, folded with the exchange of a given number of color octets (gluons) and subsequent creation of new color singlets (strings). A first (empirical) application of this approach, which we consider as very different from [7, 8], to the multiple collision process is reported in Ref. [13]. In this paper we give a concise summary of the main results of this work obtained up to now, and of some of its implications.

\section{Proton-proton and proton-nucleus collisions at CERN SPS energies}

It is quite natural to investigate the question from Section 1 using baryon spectra in the final state as experimental input. The latter, if experimentally precise enough, benefit from baryon number conservation and are particularly sensitive to the initial state of the projectile in terms of momentum (leading particle effects, Feynman scaling) and flavor content (isospin effects). Unfortunately few datasets are characterized by sufficient completeness (protons, neutrons, strange baryons) and coverage (preferably full) in rapidity. The latter requirement puts a stringent limitation on studied collision energy, precluding e.g. the LHC regime. The NA49 [14] results on pp and minimum-bias $\mathrm{pC}$ reactions at $\sqrt{s}=17.3 \mathrm{GeV}[15,16]$ constitute to the best of our knowledge the best dataset as far as the above requirements are concerned. Rapidity distributions of protons and neutrons in pp and "multiple" proton-carbon collisions ${ }^{1}$ are presented in Fig. 1. Several items are noteworthy: (1) the high-rapidity "diffractive" peak in the proton spectrum in pp collisions is absent in the neutron

\footnotetext{
${ }^{1}$ Proton-carbon collisions in which the projectile proton collides with $n \geq 2$ nucleons. For more details see the original papers $[12,13,17]$.
} 


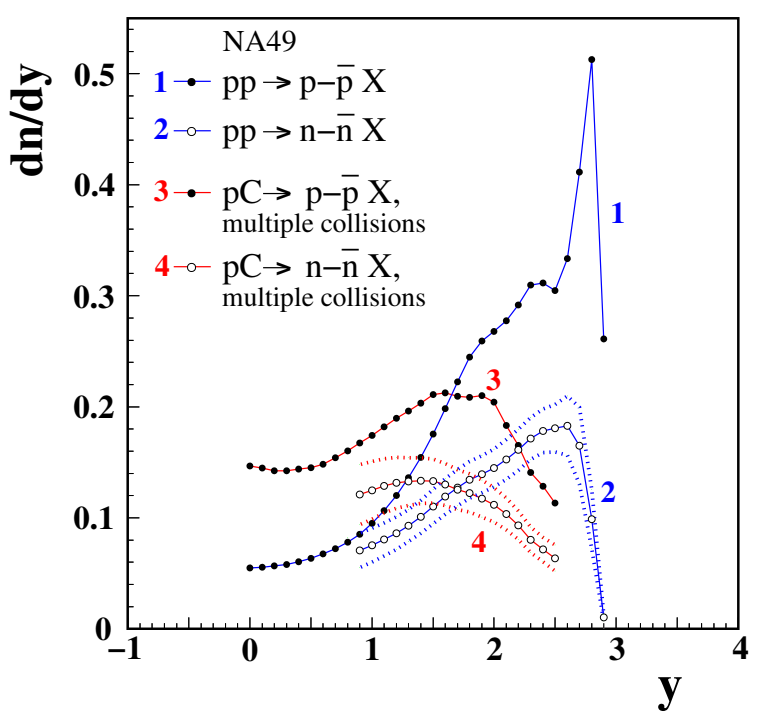

Figure 1: Rapidity distributions of net baryons $(B-\bar{B})$ in pp collisions and $\mathrm{pC}$ reactions in which the proton collides with $n \geq 2$ nucleons (the plot comes from Ref. [9] and the original NA49 data from Refs. [15, 16]).

distribution and at the same time, vanishes in multiple $\mathrm{pC}$ collisions at $x_{F}>0.9$ (a comparison of corresponding $x_{F}$ spectra can be found in Ref. [12]), (2) although the multiple pC reaction sample corresponds to an average number of only about 2.6 proton-nucleon collisions per event [18], it is characterized by a very sizable rapidity shift (baryon stopping) with respect to pp reactions, and (3) the very strong isospin effects between protons and neutrons, present at high rapidity in pp collisions, survive largely intact in the collision of a proton with multiple nucleons. These observations appeared limiting enough to serve as phenomenological guidance for our formulation of the GEM model where each soft inelastic process in the hadronic or nuclear collision occurs through the exchange of a given number of gluons between the constituent partons. A detailed account of the evolution of our understanding from the original Dual Parton Model (DPM [5, 10]) to GEM can be found in Ref. [13] and only the most important findings will be mentioned here.

\section{The Gluon Exchange Model in pp collisions}

The overall phenomenology of GEM remains similar to that of the Dual Parton Model and can be illustrated via the cartoon (a) in Fig. 2. The exchange of one soft gluon in the pp reaction changes the color configuration of constituent partons which leads to the formation of new color singlets (strings, marked as arrows in the Figure). These strings (or "chains" following the original nomenclature from [5, 10]) fragment into final state hadrons following given fragmentation functions which, for the case of final state baryon spectra, have to be tuned to experimental pp data (unlike in the earlier studies [1-8], the present dataset [15] includes protons and neutrons and therefore allows for a full treatment of isospin effects).

Importantly and unlike the DPM, GEM considers a broad even if natural spectrum of Fock states for the projectile proton (and also target nucleon). Thus already in pp collisions, the proton is to be considered as a (potential) set of a color triplet (valence quark), antitriplet (valence diquark), as well as a color triplet (sea quark) and antitriplet (sea antiquark). The general constituent momentum 
distribution follows the formula we gave in Ref. [12]:

$$
\begin{aligned}
& \rho_{m}\left(x_{q_{1}}, x_{q_{2}}, x_{q_{3}}, x_{1}, \ldots, x_{2 m}\right)= \\
& C_{m}\left(x_{q_{1}}+x_{q_{2}}\right)^{1 / 2} x_{q_{3}}^{-1 / 2} \prod_{i=1}^{2 m}\left(x_{i}^{2}+4 \mu^{2} / s\right)^{-1 / 2} \cdot \delta\left(1-x_{q_{1}}-x_{q_{2}}-x_{q_{3}}-\sum_{i=1}^{2 m} x_{i}\right)
\end{aligned}
$$

where $C_{m}$ is a normalization factor, $x_{q_{1}}, x_{q_{2}}, x_{q_{3}}, x_{1}, \ldots, x_{2 m}$ are the fractions of total momentum carried by the three valence quarks and the $2 m$ sea quarks and antiquarks (for pp collisions $m=0$ or 1), $\mu$ is the transverse mass of the sea quark, and $s$ is the square of collision c.m.s. energy.
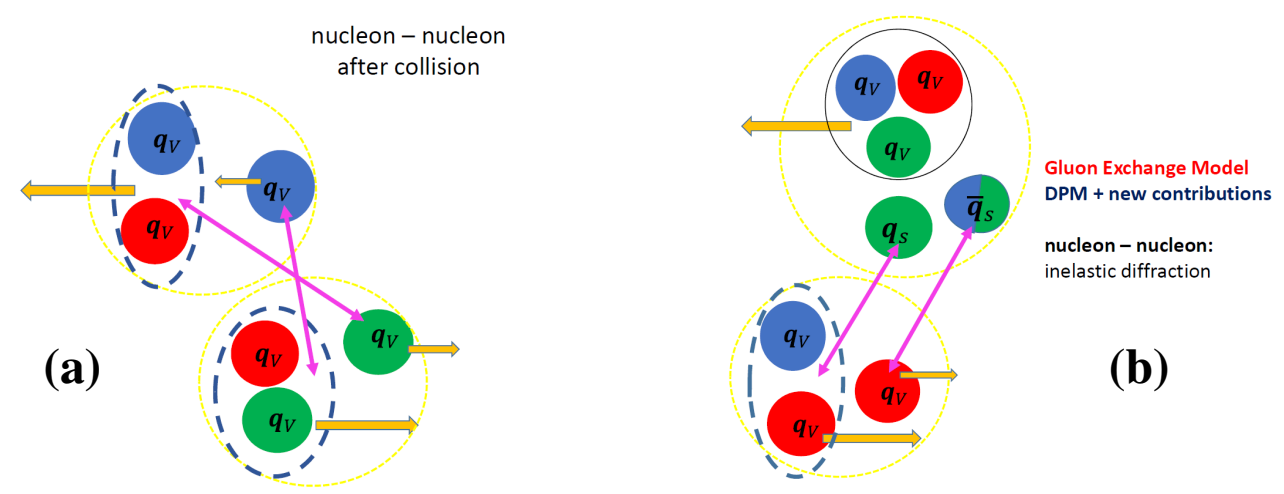

Figure 2: Basic GEM diagrams in pp collisions. Magenta arrows connect newly created color singlets (strings). The diagram (a) is inherited from the Dual Parton Model [10]. For more details, see Ref. [12].

In pp collisions, this natural extension of the Fock space leads to the appearance of an additional diagram illustrated in Fig. 2 (b), where the exchanged gluon couples to the virtual sea quark-antiquark pair. As color algebra precludes color neutralization in this $q_{s}-\bar{q}_{s}$ pair after the exchange occured, the new color singlets form in the way illustrated in the Figure keeping intact the valence structure of the final state fast proton, which explains the absence of a "neutron diffractive peak".

As it was demonstrated in Ref. [12], these two diagrams achieve an exact description of the complete final state proton and neutron spectra in inelastic pp collisions, thus falsifying the long standing opinion that the proton diffractive peak is originating from a mechanism different than color exchange [19]. At the same time, the very strong suppression of the diagram (b) in the collision of a proton with two or more nucleons explains the absence of the diffractive peak in $\mathrm{pC}$ collisions in Fig. 1.

\section{The diquark in $\mathrm{pA}$ collisions}

As it was the case for pp reactions, the inclusion of a properly complete set of Fock states leads, in proton-nucleus collisions, to a set of color exchange-induced configurations. A few examples of these are illustrated in Fig. 3 (see Refs. [12,13] for comparison). Only the diagram (a) is present in the original Dual Parton Model, while some degree of similarity exists between our diagram (b) and the "diquark-breaking" component obtained by Capella and Salgado largely based on the string 


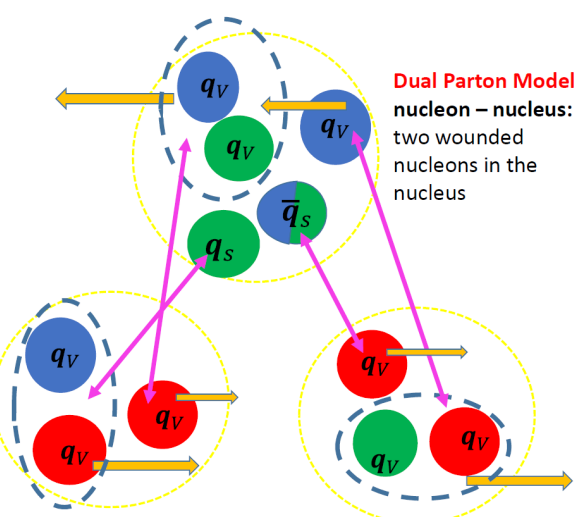

(a)

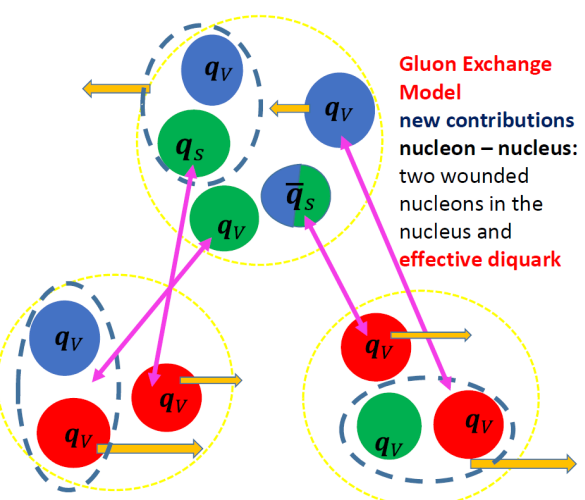

(b)

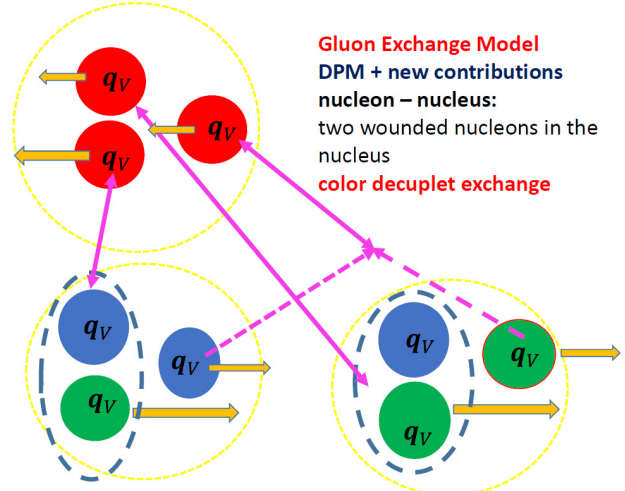

(c)

Figure 3: Examples of GEM diagrams in pA collisions for $n=2$. The diagram (a) is inherited from the Dual Parton Model [10]. For more details, see Ref. [12].

junction concept by Rossi and Veneziano (see Refs. [7, 8] and [11], respectively). Here we underline, however, the rigorous character of GEM which relies on the pure exchange of gluons rather than invoking string junction. This builds up an irrefutable difference between the two approaches with GEM following closer, in our mind, the Ockham's razor principle.

It is therefore not surprising that the diagram (c) from Fig. 3 has no counterparts in the works $[7,8]$. This diagram, resulting from a two-gluon color decuplet exchange with the valence quarks of the projectile proton, places the latter in a color-symmetric configuration. Such a configuration cannot anymore be described by a quark+diquark system.

This touches an important feature of the GEM model which is the purely effective nature of the diquark. This feature, discussed in detail in Ref. [9], places our model in opposition to the original DPM [5, 10] and, e.g., to the Wounded Constituent Model [20-22]. From the point of view of color algebra the diquark is to be seen merely as a color antitriplet configuration and as such, it can disintegrate whenever exchange with more than one color octet (gluon) occurs. Similarly as indicated by the diagram (b), a valence and sea quark can "recombine" into an effective diquark 
(color antitriplet), the fragmentation mechanism of which will be similar to that of a valence diquark-quark chain in pp collisions. It is evident that the appearance of the effective diquark built of two sea quarks is another firm prediction of GEM, a subject which we discuss in more detail in a separate paper [23]. It should be noted that all of this implies a qualitative difference between single $(n=1)$ nucleon-nucleon collisions in pp reactions and multiple $(n \geq 2)$ nucleon-nucleon collisions which occur in pA reactions (diagrams (b) and (c) cannot appear in single pp collisions).

These ideas have been tested in our papers [12,13]. Our quantitative calculation [12] with no free parameters added to the model shows that restricting the multiple collision process to the valence diquark-preserving scenario fails to reproduce the multiple collision data from Fig. 1, very significantly underestimating the "nuclear stopping power", that is overestimating baryon yields at high and underestimating baryon yields at low rapidity. The upper limit for this contribution is $48 \%$, leaving the remaining $52 \%$ for the disintegration of the valence diquark, with a possible significant contribution of the scenario with creation of an effective diquark replacing the original valence object like e.g., the $q_{v}-q_{s}$ configuration shown in diagram (b) in Fig. 3.

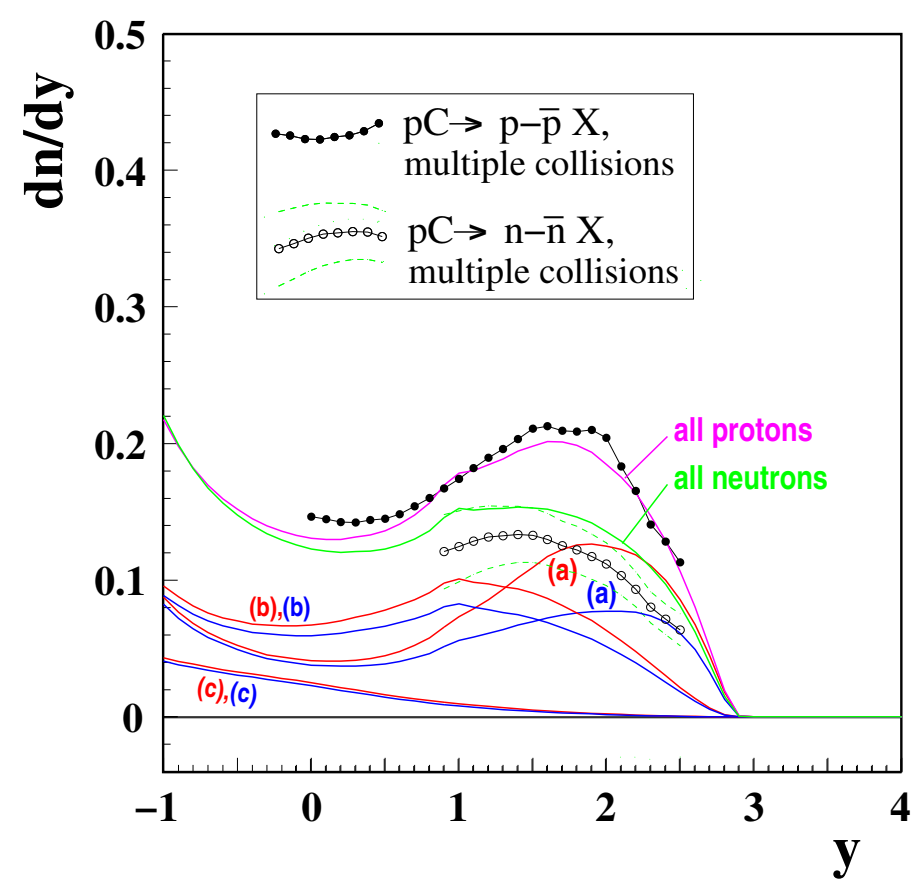

Figure 4: Description of rapidity distributions of net baryons in $\mathrm{pC}$ reactions in which the proton collides with $n \geq 2$ nucleons, by the sum of diagrams (a), (b) and (c) with respective weights $0.46,0.42$ and 0.12 . The figure is re-drawn from Ref. [13] (each diagram includes the presence of "diffractive" nucleon emission from the $\mathrm{C}$ target, see the original paper for details). The data points are the same as in Fig. 1 and the original NA49 data come from Refs. [15, 16].

Our present, state-of-the-art description of the experimental $\mathrm{pC}$ data by the diquark-preserving (Fig. 3 a), effective diquark (Fig. 3 b), and color decuplet (Fig. 3 c) contributions is presented in Fig. 4. This - still quite rudimentary - application of the GEM model to the case of $n \geq 2$ proton-nucleon collisions provides a satisfactory description of the multiple collision process in proton-carbon reactions. Keeping the valence diquark-preserving contribution close to its upper 
limit of $48 \%$ and leaving the remaining $\sim 50 \%$ to color decuplet and effective diquark diagrams provides a good description of the overall baryon nuclear stopping power and inherits most of the strong isospin (proton versus neutron) effects present in pp reactions.

\section{Conclusions}

This paper presents a short status report on our work on explaining the baryon stopping phenomenon, or the puzzle of nuclear stopping power, by a new model of pure color octet (soft gluon) exchange in $\mathrm{pp}$ and $\mathrm{pA}$ reactions.

Our results indicate that spectra of baryons are governed by color configurations of constituents, that is valence and sea quarks.

They also point at the presence of a qualitative difference between single and multiple protonnucleon collisions, that is, between pp and pA reactions. In pp collisions, inelastic baryon spectra including "diffractive" protons can be fully described by the exchange of one soft gluon coupling either to valence quarks or to the sea quark-antiquark pair. In pA reactions the exchange of two or more color octets brings new color configurations, revealing the purely effective character of the diquark which can be either disintegrated or recombined from available valence and sea quarks, which leads to stronger baryon stopping as a function of nucleus size and centrality.

The encoding of knowledge on the number of nucleons which the proton collides with proceeds through the partonic structure of the proton, account taken of a properly complete ensemble of Fock states. The increase of the nuclear stopping power in pA reactions is driven by the appearance of new color configurations with disintegration of the valence diquark and possible appearance of effective diquarks including sea quarks.

\section{Acknowledgments}

The authors kindly thank the organizers of the EPS-HEP2021 Conference. This work was supported by the National Science Centre, Poland (grant no. 2014/14/E/ST2/00018).

\section{References}

[1] M. Jeżabek and K. Zalewski, Acta Phys. Polon. B11, 425 (1980).

[2] A. Brenner et al., Phys. Rev. D26, 1497 (1982).

[3] W. Busza and A. S. Goldhaber, Phys. Lett. B139, 235 (1984).

[4] R. Bailey et al., Z. Phys. C29, 1 (1985).

[5] M. Jeżabek, J. Karczmarczuk and M. Różańska, Z. Phys. C29, 55 (1985).

[6] M. Jeżabek and M. Różańska, Phys. Lett. B175, 206 (1986).

[7] A. Capella, E. G. Ferreiro and C. A. Salgado, Phys. Lett. B 459, 27 (1999). 
[8] A. Capella and C. A. Salgado, Phys. Rev. C 60, 054906 (1999), see also: A. Capella and B. Z. Kopeliovich, Phys. Lett. B 381, 325 (1996).

[9] M. Jeżabek and A. Rybicki, Acta Phys. Polon. B 52, 981 (2021).

[10] A. Capella and J. Tran Thanh Van, Phys. Lett. B93, 146 (1980).

[11] G. C. Rossi and G. Veneziano, Nucl. Phys. B 123, 507 (1977).

[12] M. Jeżabek and A. Rybicki, Phys. Lett. B816, 136200 (2021).

[13] M. Jeżabek, A. Rybicki, Eur. Phys. J. Plus 136, 971 (2021).

[14] S. Afanasiev et al. [NA49 Collaboration], Nucl. Instrum. Meth. A430, 210 (1999).

[15] T. Anticic et al. [NA49 Collaboration], Eur. Phys. J. C65, 9 (2010).

[16] B. Baatar et al. [NA49 Collaboration], Eur. Phys. J. C73, 2364 (2013).

[17] M. Jeżabek and A. Rybicki, Acta Phys. Polon. B51, 1207 (2020).

[18] G. Barr et al., Eur. Phys. J. C49, 919 (2007).

[19] K. Fiałkowski and W. Kittel, Rept. Prog. Phys. 46, 1283 (1983).

[20] A. Białas and A. Bzdak, Phys. Lett. B649, 263 (2007).

[21] A. Białas and A. Bzdak, Phys. Rev. C77, 034908 (2008).

[22] M. Barej, A. Bzdak and P. Gutowski, Phys. Rev. C100, 064902 (2019).

[23] M. Jeżabek and A. Rybicki, arXiv:2111.03401 [nucl-th]. 